\title{
Airport Unmanned Counters Simulation Using the LabVIEW
}

\author{
Da-Un Kim ${ }^{1}$, Min-Seok Jie ${ }^{2}$ and Won-Hyuck Choi ${ }^{3}$ \\ ${ }^{1,2,3}$ Department of Avionics Engineering Hanseo University \\ ${ }^{1}$ kdu0011@naver.com, jiems@hanseo.ac.kr, ${ }^{3}$ choiwh@hanseo.ac.kr
}

\begin{abstract}
One of the important roles of the airport, the facilities for passenger service improvement, is becoming increasingly advanced. Therefore, the passengers who use the airport are making effort to provide comfortable, pleasant and timely service. As the number of passengers in the airport increases, the complexity and waiting time become longer. For this reason, foreign airports prefer unmanned counters rather than manned counters in order to streamline airport procedures. We have developed a simulator to use these unmanned counters more efficiently.
\end{abstract}

Keywords: Queuing, Unmanned Counter, Counter Simulation, LabVIEW, Airport simulation

\section{Introduction}

Aircraft are transporting travelers as new means of transportation. The role of the aircraft contribution to international or domestic industrial development in the country is increasing. Governments in each country have been able to actively positivity and develop the aviation industry, including securing airlines, modernizing airport facilities, and managing airports. One of the important roles of modern airports, the facilities for passenger service improvement, is becoming increasingly advanced. An important role of modern airports is to upgrade its facilities to improve passenger service. It is the current trend of airports to provide more convenient, comfortable and high-quality services to passengers using the airport [1-2].

However, because of the increasing number of passengers every year, the airport is becoming increasingly crowded, which is causing discomfort to passengers using the airport. In the case of domestic airports, the congestion level of airports is being lowered through the expansion of infrastructure, but this method is only a short-term countermeasure. For this reason, foreign airports are aiming at simplification the airport procedures and simplification airport operations. In order to simplify the procedure, it is more efficient to install an unmanned counter. The procedure can be simplified to reduce waiting time. It is also efficient in terms of space [3].

In general, you should use unmanned counters in the order of arrival first. This method is called first in first out (FIFO) and is referred to as a queue structure. Therefore, this paper developed an airport unmanned counter simulation tool using queuing theory and LabVIEW [4].

\section{Related Studies}

\subsection{Queuing}

A theory covering queue mathematically. Waiting to arrive at the queue, and enables mathematical and probabilistic analysis of the processes being serviced. It is a useful theory to measure performance based on the current state, based on the average latency of the system, the prediction of the service, and the estimation of the queue. The server is the 
person who carries out the service. In the case of the airport, it becomes the desk staff, but it is not necessarily the person. When a waiting person, like an unmanned counter, waits to receive a ticket, the server achieves its purpose. The following diagrams are the most basic models of the queuing system [5-6].

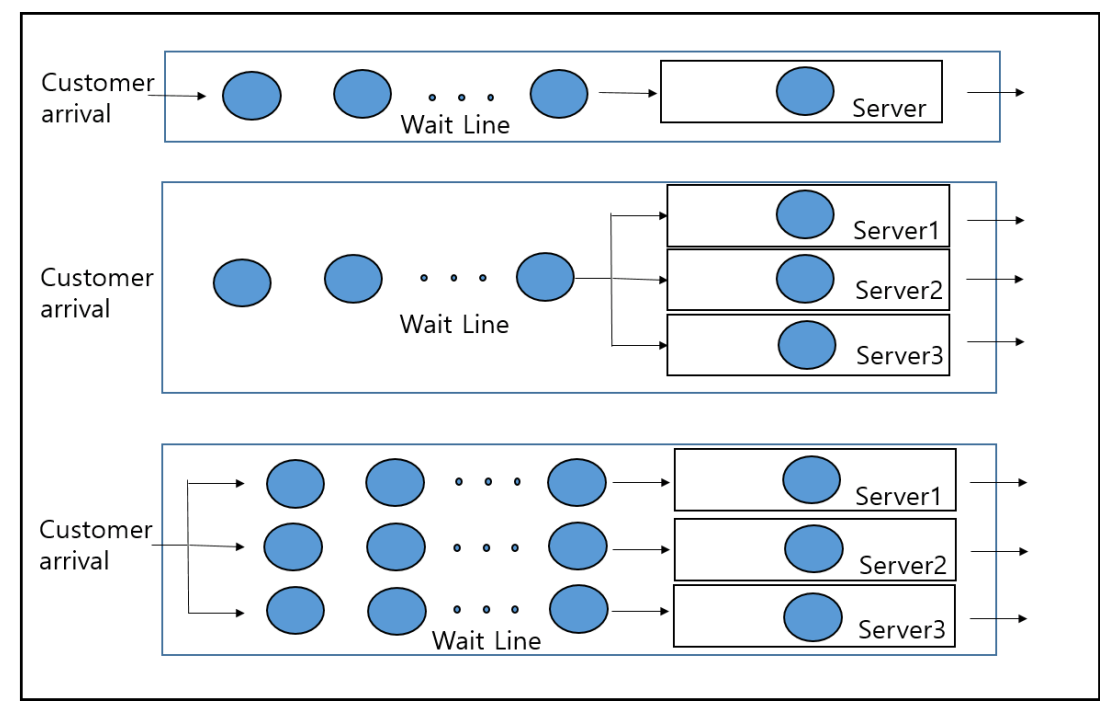

Figure 1. Basic Queuing Model

$$
\begin{aligned}
& W=\frac{1}{\mu-\lambda} \\
& \mathrm{L}=\lambda W \\
& W_{q}=W-\frac{1}{\mu} \\
& L_{q}=\lambda W_{q}
\end{aligned}
$$

$\mathrm{W}$ is the average time a guest is staying in the system (the sum of the waiting time and the service time), $\mathrm{L}$ is the average number of guests in the entire system (total waiting or receiving service), $\mathrm{Wq}$ is the average time that one customer waits to receive service, $\mathrm{Lq}$ is the average number of guests waiting to receive service [7-8].

\subsection{Unmanned}

Utilization of Drone, which has been put into dangerous places or where people cannot access it, is increasingly used for agriculture, broadcasting, and delivery. In addition to this, the Internet of Things (IoT) allows devices other than the typical communication devices, such as air conditioners and sensors, to be connected to the Internet. Robots and unmanned facilities for large-scale production Organizational Automated and unmanned, mostly used in the country, have come to life every day. IoT can operate the device regardless of the construction, and the information can be transmitted to the user through the communication technology or can be automatically integrated with the device. The subject has spread to the object as compared with the former, which is the subject who collects and processes information using objects. In the past, machines and technology have replaced many of the jobs that utilized the workforce [9].

It is an unmanned environment with advantages such as convenience, personalization and security, but it does not have merit. The unmanned environment provides services based on the results of collecting and learning personal life patterns, and cannot guarantee the security level of personal information collected in the process. Even if the highest level of security is secured at present, it is always exposed to the risk of hacking. The use of personal information may be a problem. Unmanned cameras around the city installed for security have the advantage of preventing accidents and crimes, and responding in a 
timely manner, but shooting without prior consent also implies the infringement of personal rights. There is also the possibility of invasion of privacy on the back side that the unmanned environment enhances satisfaction by providing very personal service.

\section{System Composition}

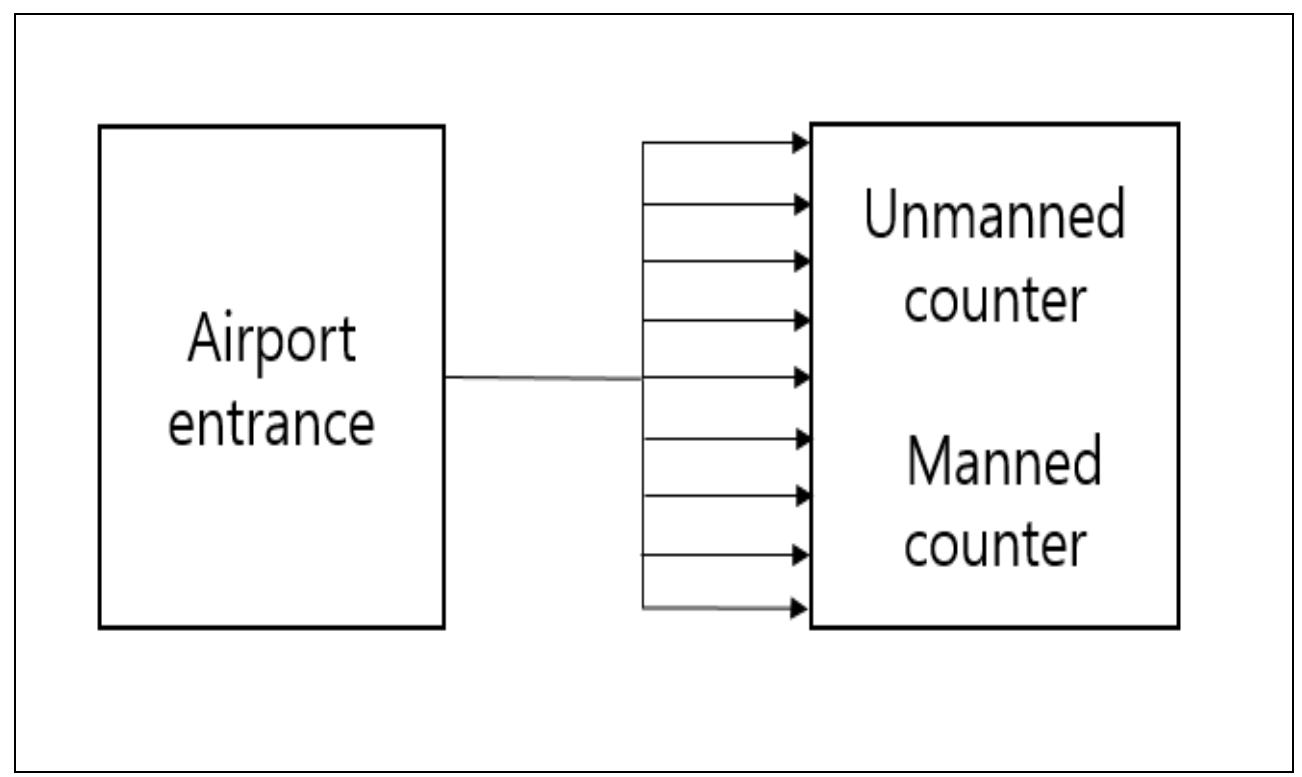

Figure 2. System Composition

In this paper, the airport unmanned counter simulation consists of a line segment from the entrance to the unmanned counter and a part where passengers operate the unmanned counter. The front panel consists of four parts. Each part is divided into data (person) generation, line image, human moving image, and graph part. For passengers to use the unmanned counter, they must use the order of the person who arrives first.

\subsection{Simulation Tool}

LabVIEW (Laboratory Virtual Instrument Engineering Workbench) is a widely used graphical programming language developed by National Instruments Inc. for testing and measurement, data acquisition and analysis, data logging, and instrument control applications. LabVIEW is the best language for developing applications related to science and engineering. Unlike text-based languages, programs are written using icons, and programs written in LabVIEW are called VI, or virtual instruments. LabVIEW uses data flow programming in which the order of execution is determined by the flow of data, unlike other programming languages in which instructions determine the order in which programs are executed. LabVIEW is easy to learn for beginners because the programming environment is graphical, allowing engineers and researchers to develop programs in a relatively short period [10].

\subsection{Airport Unmanned Counter Simulation Basic Operation and Flowchart}




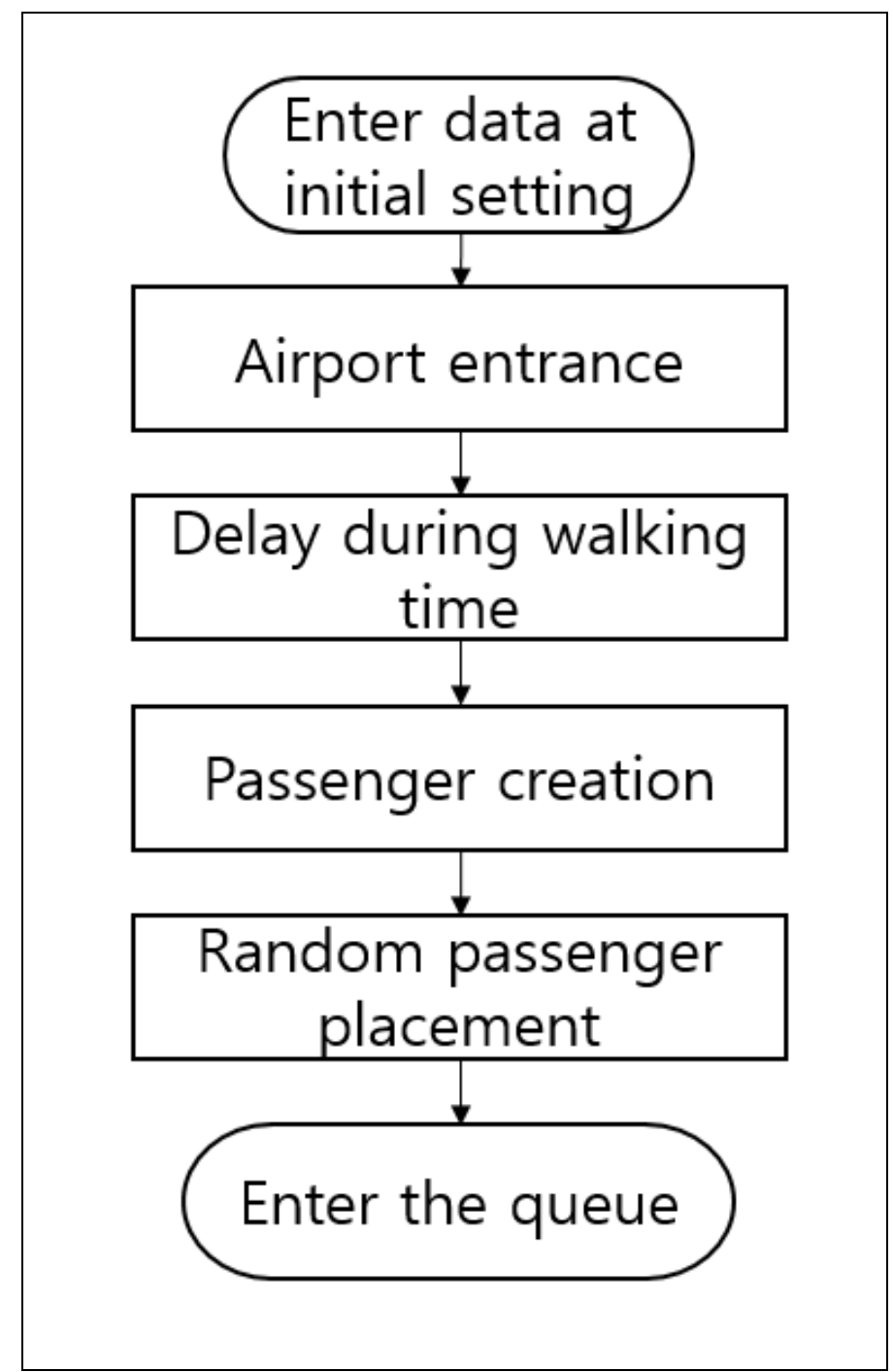

Figure 3. Flowchart to the Line at the Airport Entrance

The airport unmanned counter simulation consists of two parts, from the airport entrance to the unmanned counter and the passengers using the unmanned counter. Figure 2 shows the flow chart from the airport entrance to the unmanned counter. It is assumed that passengers come in from the airport entrance. Because there is a distance from the entrance of the airport to the unmanned counter, it is necessary to set the walking time. And you must set the time for the manned counter, the time for the unmanned counter, and the time for moving by age. It is divided into $10 \sim 20 \mathrm{~s}, 30 \mathrm{~s} \sim 40 \mathrm{~s}, 50 \mathrm{~s}$ passengers. And set the number of people to be created. An array is created in the order of 10 to 20 units, 30 units to 40 units, and 50 units. However, passengers arriving at the airport are randomly rearranged because the order is not clear. One such array is entered the queue, which becomes the line before the unmanned counter. 


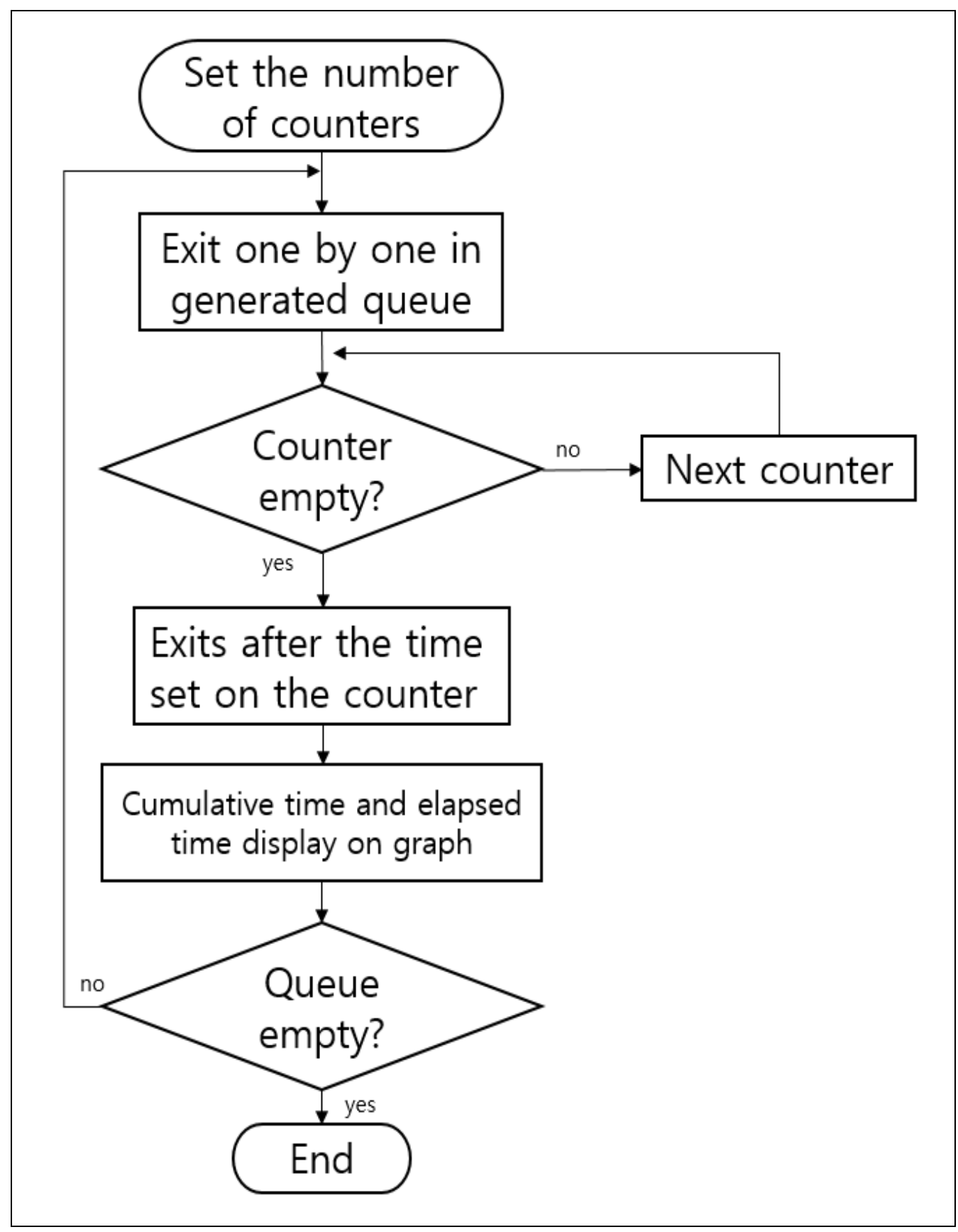

Figure 4. Unmanned Counter Flowchart

Figure 3 is a flow chart of the second part of passengers using unmanned counters. First, you must set the number of unmanned and manned counters. There are 7 unmanned counters on the simulation and 3 manned counters. You can operate as many as one to ten units. In the first part of the generated queue, the front most passenger comes out and enters the first unattended counter. The next passenger enters the next unmanned counter. The passengers who arrive in order from the queue navigate to the vacant space of the unmanned counter and enter when there is empty space. At this time, the search is performed in the order of the unmanned counter, and the search speed is 0.1 second. 


$$
\begin{gathered}
X_{a x i s}=T_{o}+T_{r} \\
Y_{\text {axis }}=T_{d_{1}}+T_{d_{2}}+T_{d_{s}}+\cdots+T_{d_{n}} \\
T_{o}=\text { Counter Operating Time } \\
T_{r}=\text { Required Time by age } \\
T_{d_{n}}=\text { Delay of } n \text {th Person }
\end{gathered}
$$

Passengers entering the unmanned counters and the manned counters come out of the unmanned counters after the 'counter operation time + time required by age'. The XY graph shows elapsed time and cumulative time. The $X$ axis shows the actual elapsed time, and the $\mathrm{Y}$ axis shows the accumulated time plus the time spent by each passenger.

\subsection{Simulation Setting}

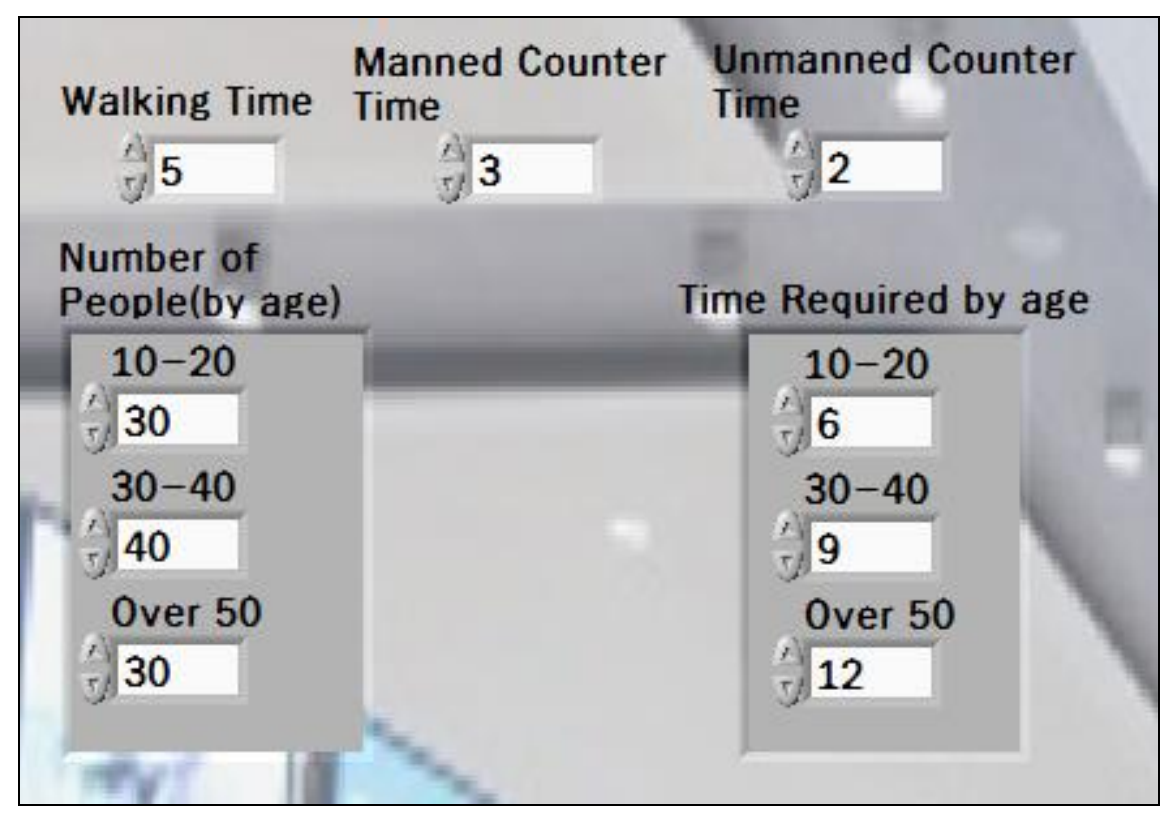

Figure 5. Initial Setting

Some configuration is required to run the airport unmanned counter simulation. You should set the number of unmanned counters and manned counters to operate, the number of manned counters to use, the number of crews by age, the time of travel by age, the time to walk from the airport entrance to the unmanned counter In the simulation of this paper, we set the time shorter than the actual time. The walking time is 5 seconds, the manned counter use time is 3 seconds, the unmanned counter operation time is 2 seconds, 10 to 20 to 6 seconds, 30 to 40 to 9 seconds, 50 to 12 seconds, and the unmanned counter Numbers were set and the simulation was performed. 


\subsection{Simulation}

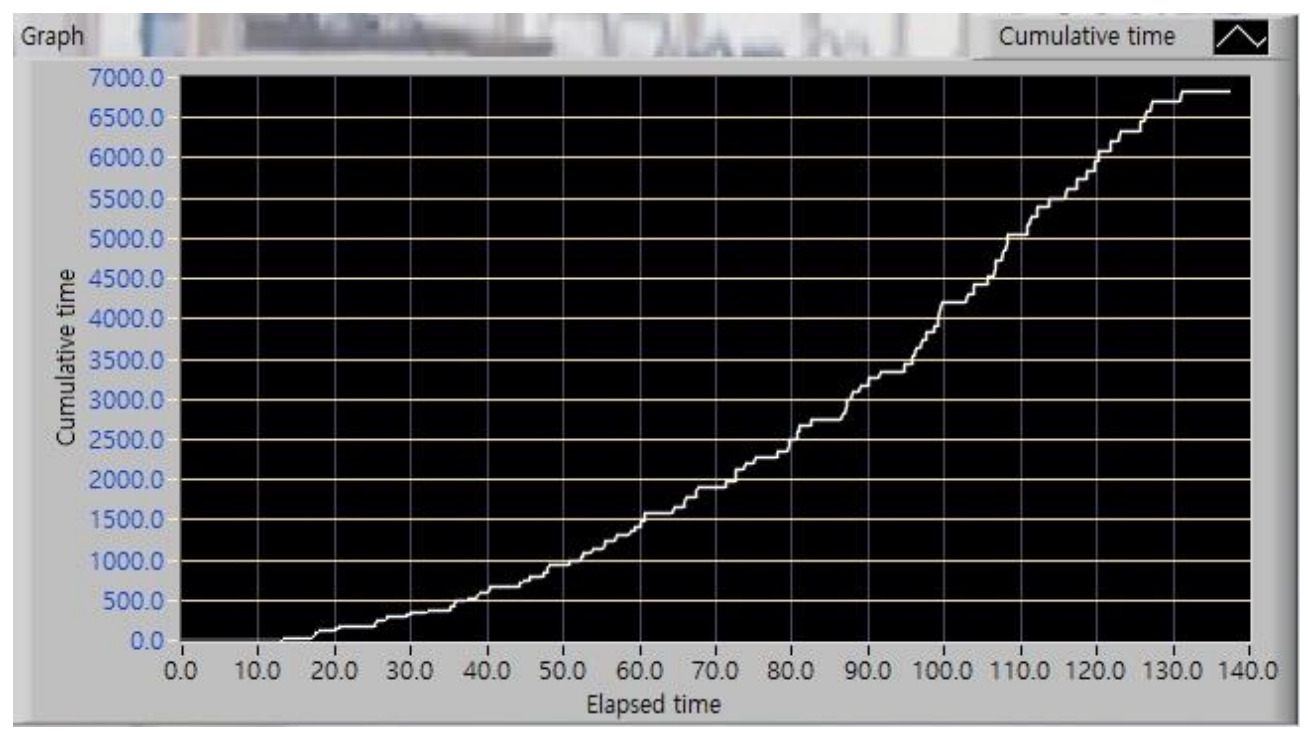

Figure 6. 10 Counter, 100 Person

In the graph in Figure 6, seven unmanned counters and three manned counters are set up. It is a graph when 30 persons in the 10th to 20th, 30th to 40th, and 30th in the 50s are set. The elapsed time of the $\mathrm{X}$ axis is 130 seconds, and the cumulative time of the $\mathrm{Y}$ axis is 6750 seconds.

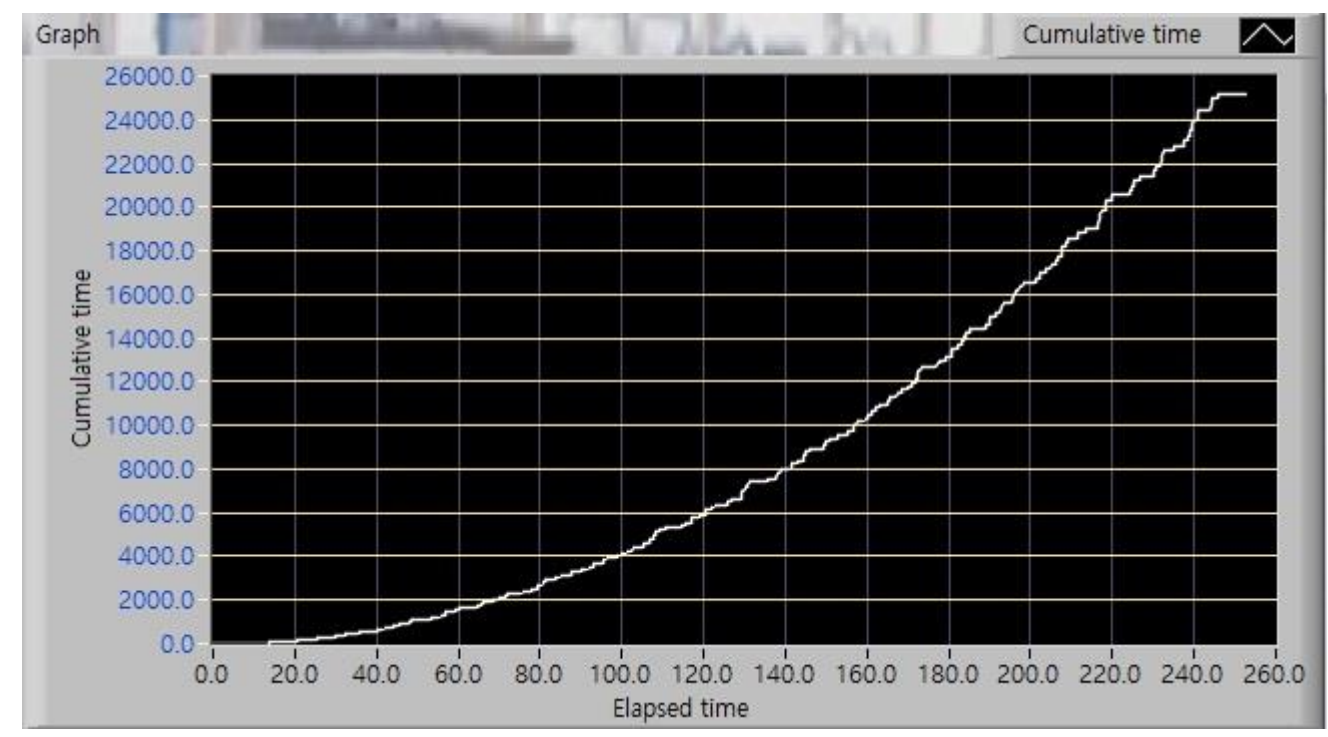

Figure 7. 10 Counter, 200 Person

In the graph in Figure 7, seven unmanned counters and three manned counters are set up. It is a graph when setting 60 persons in the $10 \mathrm{~s} \sim 20 \mathrm{~s}, 80 \mathrm{~s}$ in the 30s $\sim 40 \mathrm{~s}$, and $60 \mathrm{~s}$ in 50 s. The elapsed time of the $\mathrm{X}$ axis is 250 seconds, and the cumulative time of the $\mathrm{Y}$ axis is 25000 seconds. 


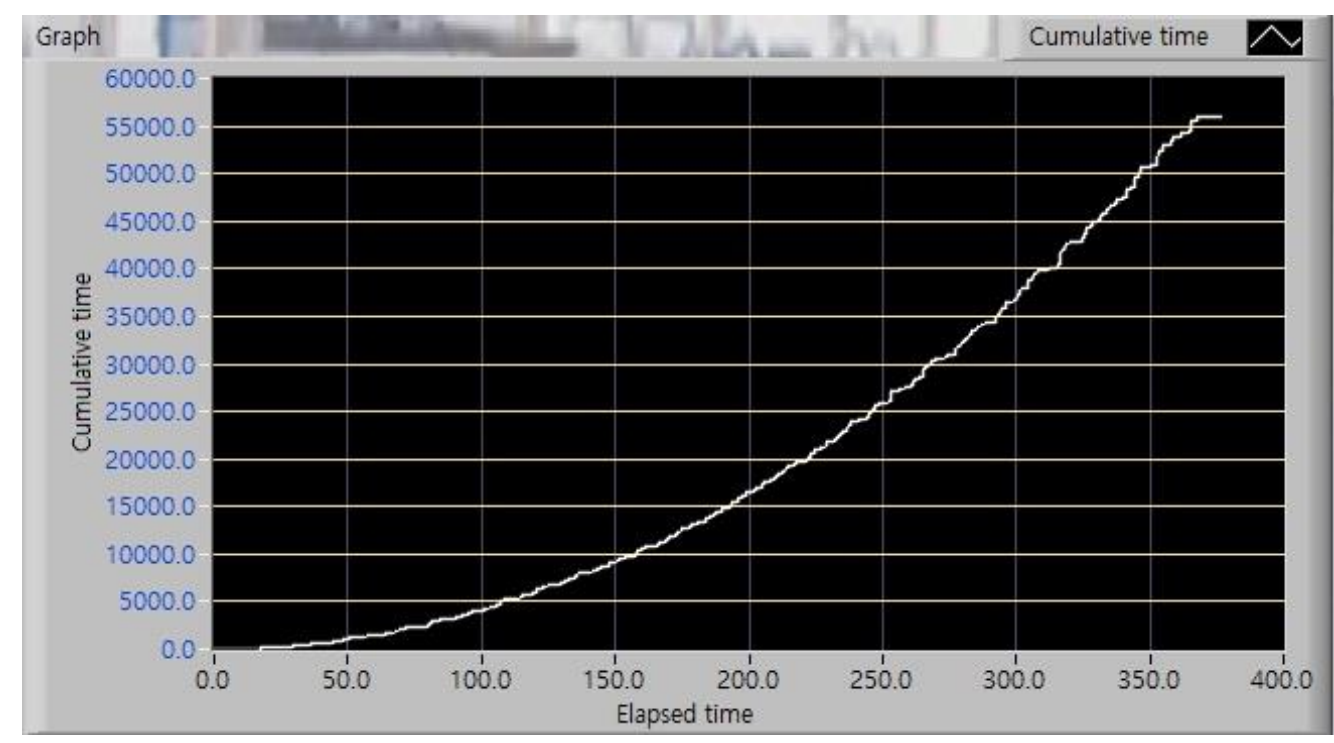

Figure 8. 10 Counter, 300 Person

In the graph in Figure 8, seven unmanned counters and three manned counters are set up. It is a graph when 90 persons in the 10s $\sim 20 \mathrm{~s}, 120 \mathrm{~s}$ in the 30s $\sim 40 \mathrm{~s}$, and $90 \mathrm{~s}$ in 50s are set. The elapsed time of the $\mathrm{X}$ axis is 370 seconds, and the cumulative time of the $\mathrm{Y}$ axis is 56000 seconds.

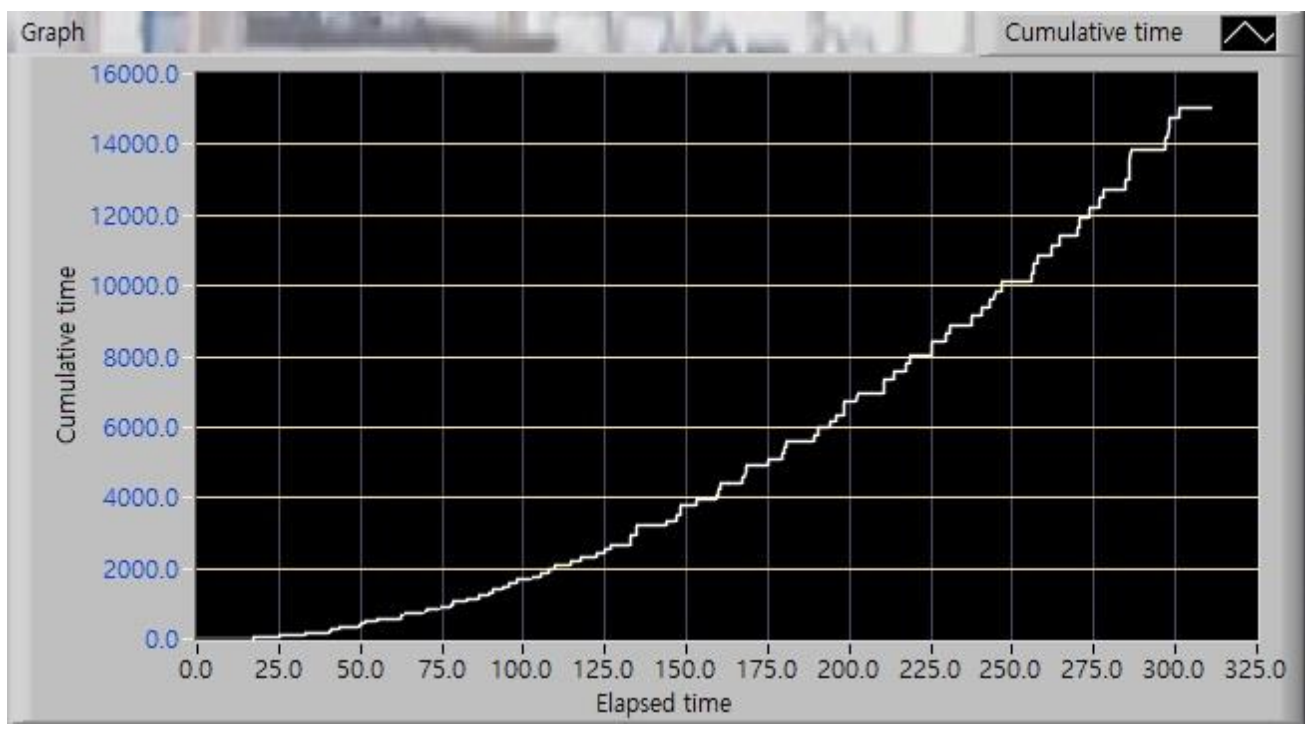

Figure 9. 4 Counter, 100 Person

In the graph in Figure 4, three unmanned counters and one manned counter were set. It is a graph when 30 persons in the 10th to 20th, 30th to 40th, and 30th in the 50s are set. The elapsed time of the $\mathrm{X}$ axis is 300 seconds, and the cumulative time of the $\mathrm{Y}$ axis is 15000 seconds. 


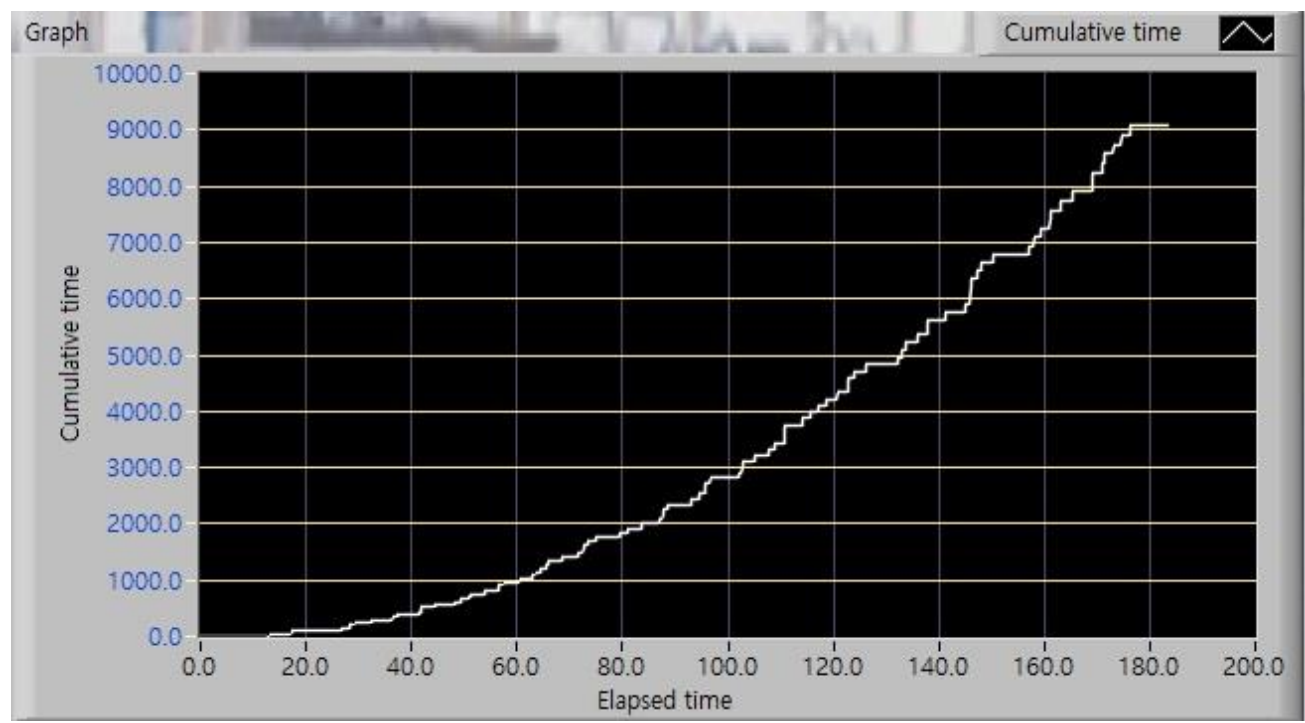

Figure 10. 7 Counter, 100 Person

In the graph in Figure 10, five unmanned counters and two manned counters were set, and a total of seven counters were set. It is a graph when 30 persons in the 10th to 20th, 30th to 40th, and 30th in the 50s are set. The elapsed time of the $X$ axis is 175 seconds, and the cumulative time of the $\mathrm{Y}$ axis is 9000 seconds.

When the elapsed times of graphs 6, 7, and 8 are compared, they are shown in the order of 130 seconds, 250 seconds, and 370 seconds. It will take 125 seconds, 245 seconds, and 365 seconds if you subtract 5 seconds from the entrance to the unmanned counter. If the passengers using the unmanned counter are doubled or tripled, the elapsed time is doubled or tripled. Also, it is possible to change the number of counters from 1 to 10 , so that simulation is possible.

\section{Conclusion}

In this paper, we developed an airport unmanned counter simulation using LabVIEW to reduce airport congestion, which is a problem of modern Airport. In the above simulation, we confirmed that this simulation can be reflected in reality. Figure 11 is a graphical representation of the unmanned counter simulation as a front panel in LabVIEW. You can see people waiting in line and moving.

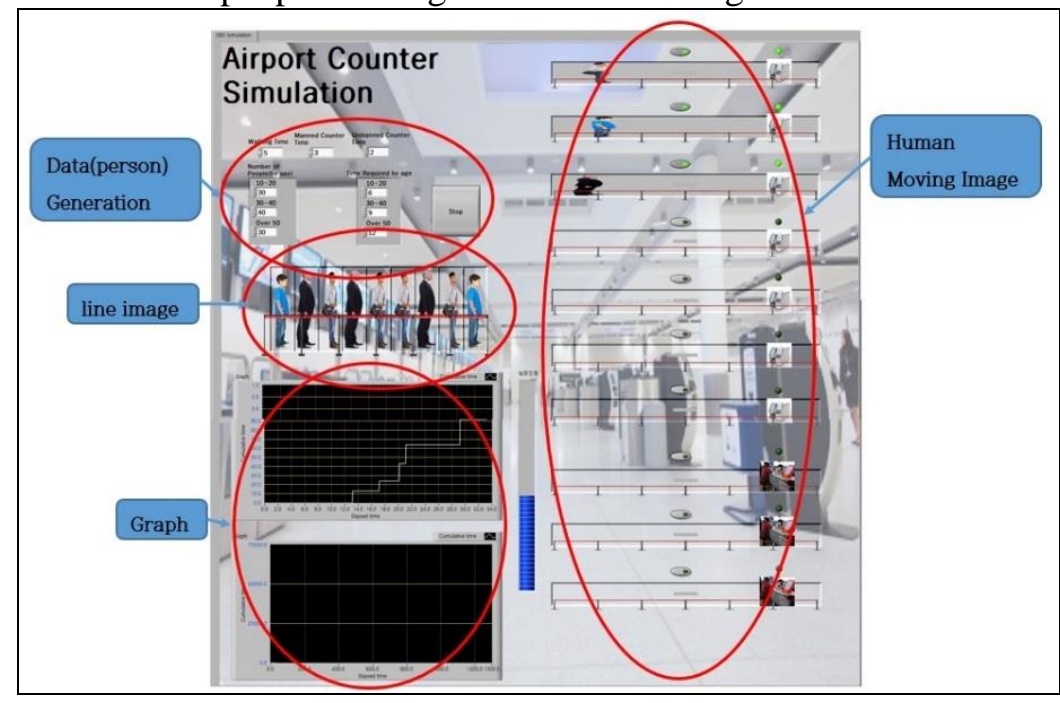

Figure 11. Development Screen 
Based on the results of the simulation, we will try to help the efficiency of the airport unmanned counter. Because simulation can be used to obtain data virtually, it is less accurate, but more efficient than setting up an actual unmanned counter to obtain data. Plans of future will reduce the error range of this simulation, extend the basic concept of the tool, and implement the concept design so that it can be applied to various fields where queuing is applied. In addition, we will develop a simulation that can simulate a process that is difficult to experiment in reality by mixing with other theories.

\section{Acknowledgement}

This paper is supported by the Intramural Research Projects Hanseo University in 2016.

\section{References}

[1] G. U. Ryu, “Airport Operation and Management”, BAEKSANS PUBLISHING, Korea, (2004).

[2] J.H. Cho and D.K. Lee, "Airport Punctuality Analysis Using Multi-Dimensional Visual Analysis Method”, Journal of the Korea Society Conference 2010 IT services., (2010), pp. 285-290.

[3] G.R. Kim and W.J. Yhang, "The Effect of the Waiting Environment on the Perceptions of the Waiting Time and the Crowding”, Korea Journal of Hotel Administration., vol. 22, no. 3, (1990), pp. 235-248.

[4] H.Y. Son, "Standard procedure of the LabVIEW", INFINITYBOOKS, Seoul, (2012).

[5] K.H. Cho, "Queuing Model for Simulation", The Journal of KIISE., vol. 8, no. 1, (1990), pp. 33-36.

[6] M. Klymash and M. Kyryk, "Data Buffering Multilevel Model at a Multiservice Traffic Service Node", SmartCR, vol. 4, no. 4, (2014), pp. 294-306.

[7] M.J. Lee and T.Y. Kim, "Adaptive Priority Queue-driven Task Allocation method for sensor data processing in IoT Environment”, Journal of Korean Institute of Industrial Engineers., (2016), pp. 62766278.

[8] C.S Kim, "Queueing System Operating in Random Environment as a Model of a Cell Operation", Industrial Engineering \& Management System., vol. 15, no.2, (2016), pp. 131-142.

[9] I.J. Jang, "Recent Research Trends and Direction of Unmanned Machine in Horticulture", Korean Journal of Horticultural Science \& Technology, vol. 31. no. S2, (2000), pp. 30-30.

[10] T.W. Kim and Y.M. Chung, "Efficient Hardware IP Control and Simulation Using LabVIEW", THE INSTITUTE OF ELECTRONICS ENGINEERS OF KOREA., (1998), pp. 514-517. 


\section{Authors}
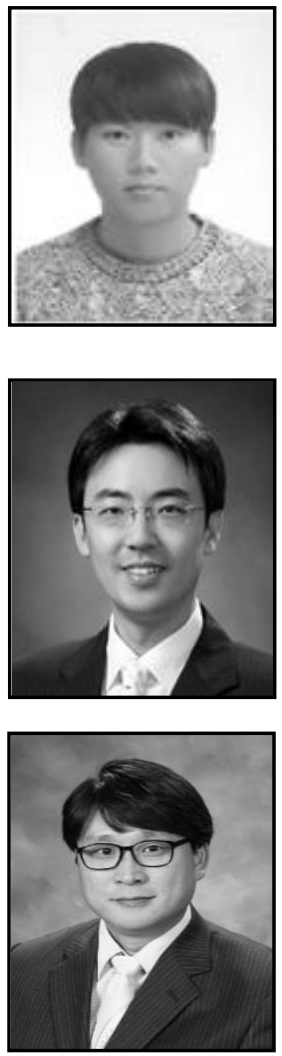

Da-Un Kim, graduate of the Hanseo University avionics bachelor's and a master's degree and Aviation Systems Engineering in 2015.

Min-Seok Jie, graduated from the Ph.d degree in the department of avionics Engineering from Korea Aerospace University in 2006. he is Hanseo University Avionics Engineering Professor in to the present in 2008.

Won-Hyuck Choi (Corresponding author), graduated from the $\mathrm{Ph} . \mathrm{D}$ degree in the department of avionics Engineering from Korea Aerospace University in 2006. the Doowon Technical University Smart Network Professor From 2008 to 2014. he is Hanseo University Avionics Engineering Professor in to the present in 2014. 
International Journal of Control and Automation Vol.10, No.4 (2017) 